\title{
Evaluation of Telomerase Activity in Nasal Polyps
}

\author{
Ernesto Bruno, M.D., ${ }^{*}$ Enzo Bonmassar, Ph.D.,\# Stefano Di Girolamo, M.D., ${ }^{*}$ \\ Riccardo Adamo, M.D.,\# Marco Alessandrini, M.D., ${ }^{*}$ Fabrizio Ottaviani, M.D., ${ }^{*}$ \\ Faustino Rosati, M.D., ${ }^{\star}$ and Ornella Franzese, Ph.D.\# (Italy)
}

\begin{abstract}
Background: The objective of this study was to assess if nasal polyps express telomerase activity and whether a difference could be found between the polyp and the surrounding mucosa of the middle meatus and between different portions of the polyp itself.
\end{abstract}

Methods: Nine patients affected by nasal polyposis were included in this study; four of these patients had recurring polyposis. Telomerase activity was measured by telomeric repeat amplification protocol assay. In six patients, the telomeric repeat amplification protocol assay was performed on the polyp and on the mucosa from the ipsilateral middle meatus. In a polyp, we were able to investigate telomerase activity of its different portions, corresponding to pedicle and fundus.

Results: Telomerase activity observed in nasal polyps was higher than that observed in samples from the ipsilateral middle meatus mucosa. High or intermediate telomerase activity was found to be related to predominant recurring polyposis.

Conclusions: Therefore, it could be postulated that telomerase activity could be related with the tendency of polyps to recur. (American Journal of Rhinology 18, 197-201, 2004)

\section{INTRODUCTION}

$\mathbf{I}^{\mathbf{n}}$ recent studies, several groups tried to identify some parameters related to the biology of nasal polyps. In particular, attempts have been made to identify differences

From the *Division of Otorhinolaryngology and \#Institute of Pharmacology, Faculty of Medicine and Surgery, University of Rome "Tor Vergata," Rome, Italy

Address correspondence and reprint requests to Ernesto Bruno, M.D., Department of Otorhinolaryngology, University of Rome "Tor Vergata," c/o Via Emilia 38, 04100 Latina, Italy Copyright (C) 2004, OceanSide Publications, Inc., U.S.A. possibly existing between polyp tissues and surrounding mucosa of the middle meatus. In this field, it could be interesting to identify some factors possibly related to the growth of polyps and to their tendency to relapse. Constant differences have been identified in the proliferation rate and in the expression of proliferating cell nuclear antigens (PCNA) of polyps with respect to sorrounding mucosa. ${ }^{1}$ Moreover, a more extensive inflammatory infiltrate in polyps with respect to the surrounding mucosa has been described. ${ }^{2}$

According to the statement that the origin and the development of polyps depend directly on the replication of the composing cellular types, we tried to investigate this condition, studying a cellular enzymatic function that was strictly related i.e., telomerase activity. Actually, telomerase is a specialized reverse transcriptase in which its function is to extend telomeres of eukaryotic cells. It is expressed in neoplastic cells, stem cells, and in selected types of growing epithelial cells, and the degree of the telomerase activity is related to the proliferation rate of the corresponding tissue. Telomerase activity also is represented in the epithelial component of benign epithelial proliferative lesions such as leucoplakia, dermatitis, and psoriasis and the degree of telomerase activity is correlated with the proliferation rate of these tissues. The chromosomes of somatic cells undergo a continuous shortening in correspondence to each mitotic division, reaching a critical length after several cell divisions described as cell senescence status. ${ }^{3}$ Therefore, telomerase could be able to guarantee an increased intrinsic replicative capacity to some actively proliferating cells. Moreover, it also is expressed in cells that are not necessarily actively proliferating. Actually, it could be detected in some inflammatory processes or in cells undergoing DNA damage repair. ${ }^{4}$

The aim of this study was to assess (a) whether nasal polyps that usually show increased proliferation index with respect to adjacent mucosa of the middle meatus, ${ }^{1}$ would be 
characterized by higher telomerase activity, (b) whether this activity possibly could be related to proliferative activation of polyp tissues and consequently to growth and/or potential recurrence of cellular components of the polyps, and (c) whether a different biological behavior could be detected between polyp tissues and surrounding nasal mucosa.

\section{MATERIALS AND METHODS}

\section{Patients and Tissue Samples}

T he study conformed to guidelines for human experimentation and was approved by the University's ethics committee. All patients provided written informed consent before their inclusion in the study.

Nine patients affected by nasal polyposis were included in this study. A detailed clinical history was obtained in order to determine the presence of conditions potentially favoring the development of nasal polyps. Four patients had recurring polyposis. One of these patients was diabetic, and one patient was allergic to dermatophagoides and showed hypothyroidism accompanied by acetyl-salicylic acid intolerance and asthma (i.e., acetyl-salicylic acid syndrome). The latter was submitted to surgical intervention four times. The remaining five patients underwent only one surgical procedure (two patients were allergic to dermatophagoides and one patient was diabetic and previously submitted for total thyroidectomy). In two out of five nonrecurrent patients, hypereosinophilia was consistently found.

The polyps were collected at the time of sinus surgery and the turbinate biopsies were performed concomitantly. Specimens of mucosa from the head and body of the middle turbinate and ipsilateral nasal polyps were taken and frozen immediately to $-80^{\circ} \mathrm{C}$. At the time of the assay (see next section), specimens were thawed and placed in complete medium composed of serum-free RPMI-1640 medium (Gibco, Paisley, U.K.) supplemented with $0.1 \%$ collagenase (Sigma-Aldrich Co., St. Louis, MO), minced in small pieces and incubated in complete medium at $37^{\circ} \mathrm{C}$ in a $5 \% \mathrm{CO}_{2}$ humidified incubator for 1 hour. Then, samples were filtered with 100-micron filters (Millipore Corp., Bedford, MA) to remove debris, and cells were collected and counted. The cell yield for each sample has always been far better than the 10,000 cells required for the telomeric repeat amplification protocol (TRAP) assay. For each sample, a fraction of proteic extract corresponding to 10,000 cells has been used for the TRAP assay. The average yield of cells obtained for turbinate mucosa was $0.5 \times 106$ and for polyps was $1-20 \times 106$.

\section{Assay for Telomerase Activity}

$\mathbf{T}$ elomerase activity was measured by TRAP assay. The TRAP assay ${ }^{5}$ works allowing the telomerase complex present in the sample to elong the trimer sequence (TS) contained in the elongation buffer, which serves as a substrate for telomerase and which functionally acts as the natural substrate of the enzyme, modified to minimize arti- facts. This phase is called elongation. At each addition, the enzyme adds to the TS sequence the characteristic ttaggg repetitive sequence and at the following addition the previously added ttaggg sequence is elongated with another ttaggg. The reaction is stopped after 20 minutes of warming the reaction to $90^{\circ} \mathrm{C}$ and denaturing the telomerase complex, which will not further elongate the TS. At this point, the wax barrier at the bottom of the reaction tube melts, freeing the polymerase chain reaction (PCR) buffer containing the p32 labeled radioactive 6-deoxycytidine-5'-triphosphate $[\alpha-32 \mathrm{P}]$ and the $\mathrm{CX}$ reverse primer. The PCR reaction is then performed with 31 cycles and radioactive dCTP is included in the PCR amplified sequence. The quantity of amplified sequences is determined by the concentration of the elongated TS, which in turn depends on the telomerase activity detected in the sample. The PCR product then is run on an acrylamide gel and the gel is exposed to an X-ray film. The signal revealed on the film depends on the concentration of radioactive PCR amplified sequences in the sample. The strength of the signal is quantified with computerized densitometry and corresponds to the degree of telomerase activity detected. The characteristic ladder pattern observed on the film is caused by the different degree of elongation that the TS sequence can undergo during the elongation time. The following PCR reaction amplifies each degree of elongation undergone by the TS. The different molecular weights of each amplified TS sequence determine a different but precise running speed on the gel. Every stair seen on the film corresponds to a length reached by a certain amount of the elongated TS. The stairs appear to be well separated because the elongation performed by the telomerase complex occurs adding at each time six nucleotides that give well-differentiated molecular weights.

Telomerase activity was assayed in whole cell extracts. Equal numbers of cells were lysed in ice-cold extraction buffer containing $0.5 \% 3[$ (cholamidopropyl)-dimethyl-ammonium]-1-propanesulfonate, $10 \mathrm{mM}$ of Tris- $\mathrm{HCl}(\mathrm{pH} 7.5)$, $1 \mathrm{mM}$ of $\mathrm{MgCl} 2,1 \mathrm{mM}$ of EGTA, $5 \mathrm{mM}$ of $\beta$-mercaptoethanol, $0.1 \mathrm{mM}$ of [4(2-aminoethyl)-benzene-sulfonyl fluoride] hydrocloride, and 10\% glycerol (Sigma). Extracts from 10,000 cells were used for TRAP assay. TRAP assay was performed in $50 \mu \mathrm{L}$ of reaction mixture $(20 \mathrm{mM}$ of Tris- $\mathrm{HCl}$ [pH 8.3], $68 \mathrm{mM}$ of $\mathrm{KCl}, 1.5 \mathrm{mM}$ of $\mathrm{MgCl} 2,1$ $\mathrm{mM}$ of EGTA, $0.05 \%$ Tween $20,0.1 \mu \mathrm{g}$ of TS [5'-AATCCGTCGAGCAGAGTT] primer, $0.5 \mu \mathrm{M}$ of T4 gene 32 protein, $10 \mu \mathrm{M}$ of deoxynucleotide triphosphate, $2 \mathrm{Us}$ of Taq polymerase [Promega, Madison, WI], and $2 \mu \mathrm{Ci}$ of (32P)dCTP [3000 CI/mmol; tube containing $100 \mathrm{ng}$ of reverse primer $\mathrm{CX}$ oligonucleotide $5^{\prime}$-(CCCTTA) ${ }_{3} \mathrm{CCC}$ TAA \{Biogen, Rome, Italy\}]) sealed at the bottom of the tube by a wax barrier. Samples were incubated at $22^{\circ} \mathrm{C}$ for 20 minutes to allow telomerase to extend TS primer, followed by a 31-cycle PCR amplification (Perkin Elmer Corp., Norwalk, CT) of the telomeric products. Forty microliters of the PCR products was run on $10 \%$ nondenaturing acrylamide gels. Gels were fixed in $0.5 \mathrm{M} \mathrm{NaCI}, 50 \%$ 


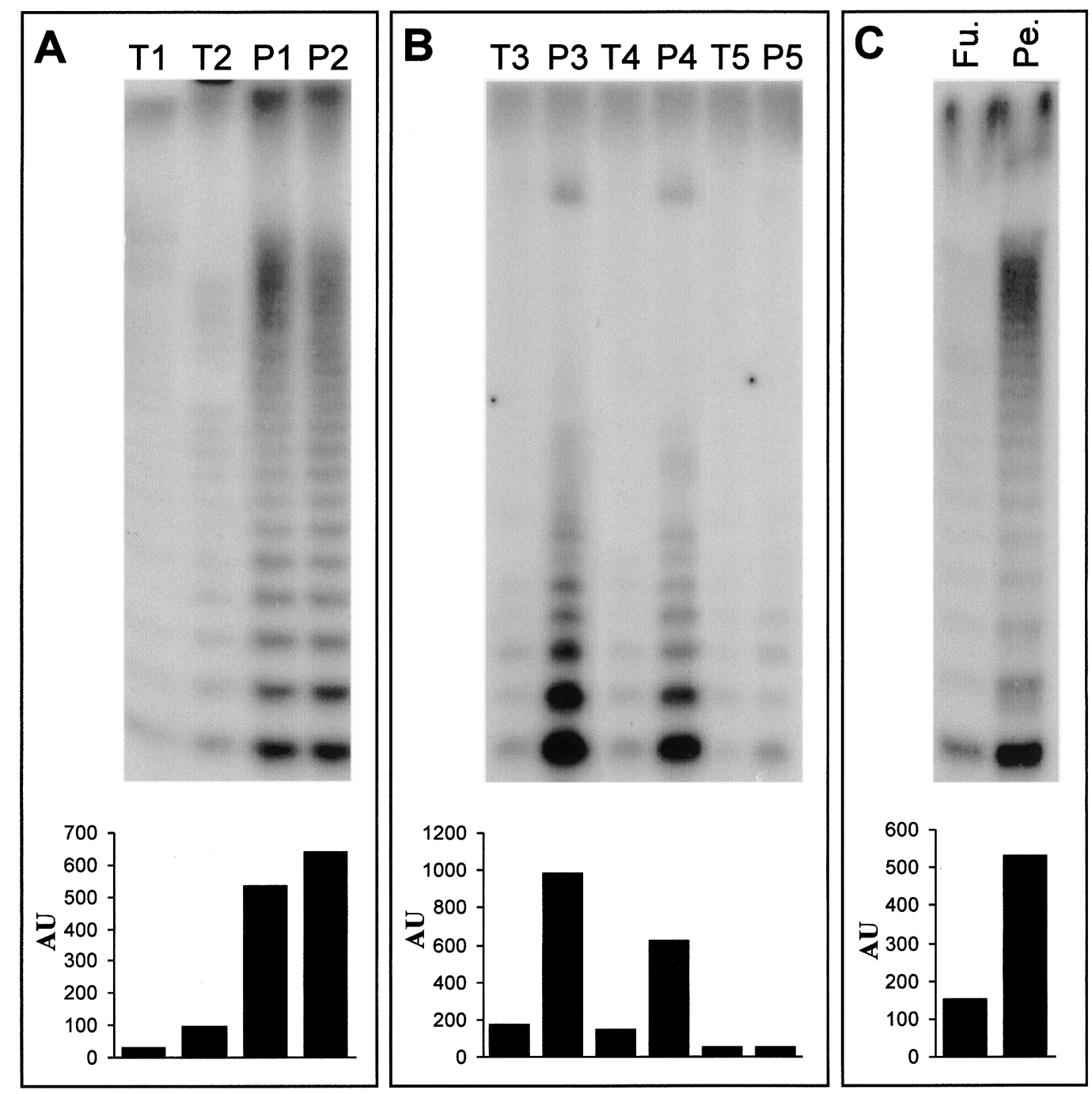

Figure 1. (A) Telomerase activity of two nasal polyps (P1 and P2) with respect to the telomerase activity of their corresponding ipsilateral turbinate (T1 and T2). Columns indicate the extent of telomerase activity expressed as "arbitrary units" (AU, see Methods section). (B) Telomerase activity of three nasal polyps with different enzymatic activity (high, P3; medium, P4; and low, P5) and corresponding ipsilateral turbinates (T3, T4, and T5). (C) Telomerase activity of two parts of the same nasal polyp (fundus Fu. and pedicle Pe.).

ethanol, and $40 \mathrm{mM}$ of sodium acetate $(\mathrm{pH} 4.2)$ and exposed to X-Ray Film (Kodak, Rochester, NY) at $-80^{\circ} \mathrm{C}$.

\section{RESULTS}

A TRAP assay of cellular components of surgically removed polyps was performed for each patient studied to determine cell-associated telomerase activity. At the same time, in six patients, the TRAP assay was performed on specimens of mucosa from the ipsilateral middle meatus and/or middle turbinate. The results, illustrated in Fig. $1 \mathrm{~A}$, show that telomerase activity in specimens from nasal polyps was higher than that observed in samples from the ipsilateral middle meatus mucosa. However, different levels of telomerase activity were found in the samples under investigation. A representative TRAP assay of nasal polyps expressing different degrees of telomerase function is shown in Fig. $1 \mathrm{~B}$. This figure illustrates the enzymatic activity of specimens taken from three different polyps expressing high, medium, or low telomerase activity, compared with that associated with ipsilateral turbinate samples. It should be noted that in all the cases the activity of polyps was higher than that observed in specimens from turbinates. Moreover, the enzymatic function of a given turbinate was more appreciable when telomerase activity observed in cells from the corresponding polyp was elevated.

We tried to find out a correlation between telomerase activity of nasal polyps and other clinical and anamnestic conditions of patients. In particular, the possibility that telomerase activity could be related with the tendency of polyps to recur was taken into consideration. In our samples, high or intermediate telomerase activity was found to be related to predominant recurring polyposis after surgery. On the other hand, low levels of enzymatic activity have been found mainly in nonrecurring polyposis. In particular, 
in the group of recurring polyposis (four patients), three patients showed high/intermediate telomerase activity, and in nonrecurring polyposis group, three of five patients showed polyps with low telomerase activity, being the other two cases associated with intermediate activity. Moreover, a moderate telomerase activity was detected in a specimen taken from a maxillary sinus polyp (data not shown).

In a polyp of remarkable dimensions, we were able to investigate telomerase activity of its different portions, corresponding to pedicle and fundus. The result of this study showed a barely detectable telomerase activity in the fundus and a highly detectable activity in the pedicle (Fig. 1C).

\section{DISCUSSION}

$\mathbf{I}$ is well known that nasal polyps can be divided histologically into four classes on the basis of their cellular composition and different types of stroma. ${ }^{6}$ The first class includes chronic inflammatory edematous polyps highly infiltrated by eosinophils with seromucous glands hyperplasia and polyps with stromal atypies. This class is absolutely the most frequent, representing $\sim 80 \%$ of all polyps. They show lining respiratory epithelium associated with a "goblet cell" hyperplasia, edematous stroma with many eosinophils, mast cells, and few fibroblasts. The second class of polyps ( $\sim 10 \%$ of total) is characterized by a substantial absence of goblet cell hyperplasia in the lining epithelium and even less stromal edema. Epithelium frequently shows a cuboidal and/or squamous metaplasia. Inflammatory infiltration is conspicuous and mostly characterized by the presence of lymphocytes mixed with fewer eosinophils. Moreover stroma contains many fibroblasts and frequently shows evidence of fibrosis. The third class ( $\sim 5 \%$ of total) is characterized by the presence of many seromucosal glands, frequently distributed in an edematous and weary stroma. The fourth class $(0.5 \%$ of total) is characterized by peculiar and atypical stromal cells, which represent "reactive" fibroblasts.

Many reports show that cells composing nasal polyps proliferate more than those of surrounding nasal mucosa. Moreover, the existence of polyps with a different cell replication rate has been described consistently. ${ }^{7}$ In all cases, the rate of replicating cells in connective and epithelium components is similar. ${ }^{8}$ Moreover, a substantial replicative activity can be observed also in inflammatory cells, which infiltrate nasal polyps. ${ }^{2}$ Probably, these different cell types behave in distinct ways according to various evolutive phases of polyps. During the development of polyps, cellular replication would be of relevant entity: in contrast, a well-developed polyp, eventually "compressed" by surrounding structures, ${ }^{9}$ would be characterized by a low hematic flow and by a low replicative potential of cellular components. ${ }^{10}$ Although many controversies exist about leukocyte infiltration of polyps, Morinaka and Nakamura indicated that cell populations of nasal polyps do not contain significantly higher lymphocyte infiltration with respect to normal nasal mucosa. ${ }^{2}$ However, they report higher num- bers of eosinophils, neutrophils, and plasmacells in polyps with respect to normal epithelium. ${ }^{2}$

Coste et al., ${ }^{1}$ compared the proliferative rate of epithelial cells from polyps with that from nasal mucosa in patients affected by nasal polyposis. The percentage of mitotic cells, as well as the expression of proliferation antigen (i.e., PCNA), were higher in polyps compared with surrounding mucosa. ${ }^{1}$ These observations show that the proliferative rate of cells composing nasal polyps is higher if compared with that of cells from nasal mucosa. Such difference could be explained by the presence of growth factors and cytokines produced by inflammatory cells infiltrating nasal polyps. Moreover, many reports emphasize the importance of fibroblast proliferation in determining the polyp growth rate. ${ }^{8,11}$ Even this cell type was endowed with a high proliferative index and a pronounced PCNA expression, similar to those observed in epithelial cells. ${ }^{8}$ A previous report showed a relationship between cell proliferative rate and the tendency of polyps to recur. ${ }^{7}$

We investigated whether nasal polyps express telomerase activity and, if so, whether a difference could be found between the polyp and the sorrounding mucosa of the middle meatus and between different portions of the polyp itself. The first observation was that telomerase activity can be found in almost all polyps and that this activity seems to be much higher than that expressed in specimens taken from nasal mucosa. However, it was observed that telomerase activity expressed by polyps was variable, as reported in the results section. Specimens from nasal mucosa were always found to express a lower telomerase activity with respect to that expressed by cells from the corresponding polyps. In particular, very low telomerase levels were found in cells from mucosae corresponding to polyps expressing low telomerase activity and a moderate telomerase function was found in mucosae sorrounding polyps expressing very high telomerase activity. It could be hypothesized that the different telomerase activity observed in polyps could be related to the type, the proliferative potential in the different portions (e.g., pedicle versus fundus), and to the age of the polyps. Telomerase activity observed in specimens from mucosae could be referred to the permanence of an appreciable inflammatory activation of the middle meatus district, typical of diseases characterized by polyps with high replicative capacity. We also evaluated telomerase activity on different portions of the same polyp. Cells from the pedicle showed a much higher telomerase activity when compared with cells derived from the fundus. The higher telomerase activity expressed by the pedicle compared with the fundus could be related to a higher proliferative rate in the pedicle, necessary for the growth of the polyp.

Coste et al. ${ }^{12}$ showed that epithelial cells of nasal polyps kept in culture with transforming growth factor (TGF) $\beta$ release vascular endothelial growth factor (VEGF), a cytokine able to promote a process of local angiogenesis, required for the growth of polyps. During the 1st days of culture "in vitro," these cells where shown to release VEGF 
even when not exposed to TGF- $\beta$. This probably was caused by the previous "in vivo" exposition to TGF- $\beta$. Moreover, an immunohistochemical study showed that cells expressing TGF- $\beta$ are well represented in nasal polyps, and that they are significantly more numerous in the pedicle than in the fundus. ${ }^{11}$ The myofibroblasts, recruited and activated by TGF- $\beta$ are, accordingly, more numerous at the level of the pedicle and they contribute to the growth of the nasal polyps inducing extracellular collagen matrix accumulation. ${ }^{13}$ The release of VEGF induced by local TGF- $\beta$ would justify the vascular component, which develops in the stroma of nasal polyps, and it is to be related to the local concentration of TGF- $\beta$. Assuming a correlation between local VEGF concentration and the angiogenic effect, angiogenesis would occur better at the level of the pedicle showing a faster growth at this place. In fact, the growth of the polyps, like the growth of any tissue bigger than $1 \mathrm{~mm}$ in diameter, can only take place if angiogenesis, dependent on VEGF, is present. So, we give support to the hypothesis that the growth of the nasal polyp takes place especially at the level of the pedicle. ${ }^{11,12}$

It must be pointed out that in the case of colon polyps, a relationship was found between telomerase activity, neoplastic transformation, and progression of colorectal carcinoma. ${ }^{14}$ However, this did not seem to be the case with nasal polyps, because no evidence of malignant transformation of the polyps described in this study was obtained so far.

According to the results shown in this preliminary study, we can hypothesize that the different telomerase activity expressed by nasal polyps could be related to the type of polyp, the cellular proliferation rate, and the tendency to recur. Now, we are investigating the involvement of the different cell types composing the structure of nasal polyps in determining the observed telomerase activity.

\section{REFERENCES}

1. Coste A, Reteau JG, Roudot-Thoraval F, et al. Increased epithelial cell proliferation in nasal polyps. Arch Otolaryngol Head Neck Surg 122:432-436, 1996.

2. Morinaka S, and Nakamura H. Inflammatory cells in nasal mucosa and nasal polyps. Auris Nasus Larynx 27:59-64, 2000.

3. Blackburn EH. Structure and function of telomeres. Nature 350 : 569-573, 1991.

4. Igarashi $\mathrm{H}$, and Sakaguchi $\mathrm{N}$. Telomerase activity is induced in human peripheral B lymphocytes by the stimulation to antigen receptor. Blood 89:1299-1307, 1997.

5. Wright WE, Shay, JW, and Piatyszek MA. Modifications of a telomeric repeat amplification protocol (TRAP) result in increased reliability, linearity and sensitivity. Nucleic Acids Res 23:3794-3795, 1995.

6. Davidson, A, and Hellquist HB. The so-called "allergic" nasal polyp. ORL J Otorhinolaryngol Relat Spec 55:30-35, 1993.

7. Bruno, E. Mohamed EI, Alessandrini M, et al. Long-term follow-up of cellular proliferation as a predictive index for the relapse of nasal polyposis. Am J Rhinol 16:237-241, 2002.

8. Nakagawa, T, Yamane, H., Nakai Y, et al. Comparative assessment of cell proliferation and accumulation of extracellular matrix in nasal polyps. Acta Otolaryngol Suppl 538:205-208, 1998.

9. Paludetti G, Maurizi M, Tassoni A, et al. Nasal polyps: A comparative study of morphologic and etiopathogenetic aspects. Rhinology 21:347-360, 1983.

10. Hassid S, Degaute MP, Dawance S, et al. Determination of proliferative activity in nasal polyps. J Clin Pathol 50:923-928, 1997.

11. Wang QP, Escudier E, Roudot-Thoraval F, et al. Myofibroblast accumulation induced by transforming growth factor-beta is involved in the pathogenesis of nasal polyps. Laryngoscope 107: 926-931, 1997.

12. Coste A, Brugel L, Maitre B, et al. Inflammatory cells as well as epithelial cells in nasal polyps express vascular endothelial growth factor. Eur Respir J 15:367-372, 2000.

13. Evans RA, Tian YC, Steadman R, et al. TGF-beta1-mediated fibroblast-myofibroblast terminal differentiation-The role of Smad proteins. Exp Cell Res 282:90-100, 2003.

14. Boldrini L, Faviana P, Gisfredi S, et al. Evaluation of telomerase in the development and progression of colon cancer. Int $\mathrm{J}$ Mol Med 10:589-592, 2002. 